\title{
Performar el archivo
}

\section{Performing the Archive}

Rocío ZAmora SAUma [rzamorasauma@gmail.com]

Freie Universität Berlin, Alemania

\section{RESUMEN}

A partir de la performance de Coco Fusco intitulada "Words May Not Be Found" (Berlin, 2017) y desde el concepto de "campo expandido" (expanded field) de Rosalind Krauss (1979) y de la crítica de Rebecca Schneider a la noción de inmediatez de la performance, este texto pretende mostrar algunos de los componentes que permiten pensar "la documentación" de la performance en video como ese medio donde se expande el campo de la performance a partir del del teatro y del video.

\section{Palabras Claves}

Performance; archivo; genocidio; audiencia; futuro

\begin{abstract}
Departing from Coco Fusco's performance entitled "Words May Not Be Found" (Berlin, 2017), as well as taking into consideration Krauss's concept of expanded field and Rebbeca Schneider's critique of the notion of immediacy of performance, this text discusses "the documentation" of performance through video. This is approached as the medium that expands the field of performance into the future and into an "alive" dimension of the archive through theater and video.
\end{abstract}

\section{KEY WORDS}

Performance; archive; genocide; audience

RECIBIDO 2017-12-01; ACEPTADO 2018-09-07

El video de la performance fue consultado en el sitio web https://vimeo.com/224666687 Agradezco a Coco Fusco por haberme facilitado la documentación de la pieza presentada en Berlín. La performance se llevó a cabo el 11 de marzo del 2017. 
What do the dying bodies of the past-the dying bodies of Hiroshima-have to do with the living bodies

of the present? And what is the role of our seeing

in establishing a relation between these two sets of bodies?

Cathy Caruth, Literature and the Enactment of Memory

Coco Fusco fue invitada a presentar una performance ${ }^{1}$ sobre el tema del lenguaje en los Kunst Werke's Weekends Series, como parte de la exposición del artista sudafricano Ian Wilson, en la Sophiensoele de Berlín. Su performance Words May Not Be Found consiste en la lectura de algunos fragmentos $^{2}$ de The Blue Book ${ }^{3}$ (1918), reporte británico que denuncia la crueldad de los alemanes en Namibia a partir de una serie de intercambios epistolares, testimonios y documentos legales. Se trata de un documento geopolítico de suma importancia donde se exponen las circunstancias del primer genocidio del siglo XX (1904-1908).

Coco Fusco es una artista cubano-estadounidense que ha trabajado ampliamente las formas de representación coloniales. La performance que realiza junto a Guillermo Gómez-Peña, The Couple in a Cage: Two Amerindians Visit the West (1992-1994) nos brinda un método de escenificación donde la performance funciona como un dispositivo que permite la instalación pública de las estructuras de representación y afección: "The cage became a blank screen onto which audiences projected their fantasies of who and what we are" (Fusco 1988: 152). Esta jaula funciona como un paradigma donde se muestra el dispositivo propio de lo teatral y, en términos generales, de la pantalla, como espacio de (auto) proyección colectiva. Esta noción de proyección, de extensión corporal, mediatizada por las imágenes que nos afectan desde el dispositivo teatral y cinematográfico permiten extender el campo temporal y espacial de estos dispositivos.

En este texto, quiero partir del concepto de "campo expandido" (expanded field) con el cual Rosalind Krauss (1979) describió hacia el final de los años 70 la expansión material e ideológica de los límites del campo de la escultura durante el siglo $\mathrm{XX},{ }^{4}$ con el objetivo de cuestionarme

1 Se retoma la diferencia entre la performance y el performance que realiza Diana Taylor (2003) con el objetivo de marcar este ámbito de prácticas que se inscriben dentro del contexto de las Artes (la performance) y prácticas que pertenecen al ámbito general de la cultura (el performance).

2 Coco Fusco realiza la selección de los textos, los cuales se citan al final de los créditos de la documentación en video de la obra presentada en Berlín y son mencionados a continuación: E.H.M. Gorges (Administrator of the conquered territory for the Union of South Africa) Report on The Natives of South-West Africa and their Treatment by Germany (1918), a/k/a The Blue Book; Friedrich Fabri, Does Germany Need Colonies? 1879; Diary and Letters of Nama Chief Hendrik Witbooi, 1884-1894; Kurd Schwabe's With Sword and Plow in German-South-West Africa: Four Years of War and Travelling, 1899; Theodor Gotthilf Leutwein - Elf Jahr Gouverneur in Deutsch-Südwestafrika (1908); Karl Dove, Die deutschen Kolonien, (1909).

3 El reporte fue oficialmente intitulado en 1918 "Report on the Natives of South-West Africa and Their Treatment By Germany”. Este reporte fue publicado por la Oficina administrativa del Parlamento británico en Windhuk (África del Este) y en London por la Oficina de documentación de la Majesty's Stationery Office en 1918. La portada de la version publicada dice: "To be purchased through any Bookseller or directly from H.M. Stationery Office at the following addresses Imperial House, Kingsway, London, W.C.2, and 28, Abingdox Street, London, S.W.1. 37, Peter Street Amnchester; 1, St. Andrew's Crescent, Cardiff; 23, Forth Street, Edinburgh; or from E. Ponsonby, Ltd., 116, Grapton Street Dublin.”

4 En el caso del modernismo, este campo expandido se dio gracias a la operación "in relation to this loss of site, producing the monument as abstraction, the monument as pure marker or base, functionally placeless and largely selfreferential." (Krauss 1979: 34). Y, en el caso del posmodernismo, se organizará "instead through the universe of terms that are felt to be in opposition within a cultural situation" (43). 
dónde opera la expansión del campo de las realizaciones escénicas ${ }^{5}$ y de la performance en particular.

Para abordar mi argumento, seguiré principalmente ciertas ideas de Rebecca Schneider (2011) en su texto Performance Remains a partir de la performance de Coco Fusco, Words May Not Be Found (Berlín, 2017). Iniciaré señalando algunos aspectos formales de esta pieza en relación con ciertos niveles de documentación que revelan procesos de memoria colectiva (Halbwachs 1997) y cultural (Assmann 2008), para seguidamente retomar algunos argumentos que se han esgrimido dentro de la disciplina de los Performance Studies sobre la relación entre archivo y performance con el objetivo de abrir ciertas preguntas que la performance de Coco Fusco pone en escena. Quiero referirme principalmente a la oposición que se ha establecido entre archivo y performance dentro de los Performance Studies, ${ }^{6}$ con el objetivo de proponer que la grabación de la performance que tiene lugar "en vivo" supone una rematerialización de las realizaciones escénicas, donde se da una multiplicación de lugares y donde se ubica precisamente la extensión de su campo a partir de la producción de otras audiencias en otros espacios.

\section{Words May Not Be Found}

El intercambio epistolar entre el jefe de la tribu Namaqua, Hendrick Witbooi y el jefe de la tribu del pueblo de Herero, Kamaharero y entre Witbooi y el gobernador Leutwein traducen las luchas de poder y la defensa encarecida por mantener la independencia que Witbooi reclama respecto de la propiedad y los derechos que le pertenecen como jefe de la Tribu frente al poder imperial y frente a la renuncia de independencia de su homólogo de la tribu Herero. Los testimonios relatan las atrocidades vividas durante el proceso genocida. Hacia el final de la performance, los/as otros/ as lectores/as abandonan el escenario y Coco Fusco lee el documento oficial de la Resolución de Asamblea Legislativa del Sudoeste de África donde no solo se prohíbe la reproducción de todas las copias de The Blue Book, sino que también se mandan a destruir todas las copias de los archivos oficiales y de las bibliotecas. ${ }^{7}$

La sobriedad del montaje, su luz tenue, sus manteles negros, la inscripción del podio y la seriedad en los rostros de sus lectores configuran el cuadro dentro de una atmósfera fúnebre que toma elementos de las prácticas rituales de un Tribunal donde se vuelven a poner en escena, citar e interpelar las distintas narrativas sobre lo acaecido, presentando los documentos a partir de la tensión de las dos partes. La organización espacial en esta performance se encuentra dividida en al menos cuatro partes con diversas funciones. Al fondo se instala la proyección de los títulos de los documentos que son leídos por el elenco. En el centro, el podio, y a su lado, dos mesas largas

5 Evitaremos referirnos con el término de "obra” a las realizaciones escénicas para alejarnos de ciertas ideas propias del contexto fundacional de las artes. El concepto proviene Erika Fischer-Lichte: "la realización escénica [es] la que determina la relación entre actores y espectadores y la que crea distintas posibilidades de movimiento y percepción, que es la que genera la espacialidad" (2011: 225).

6 Phelan (1996), Auslander (1999), Taylor (2003, 2009), Schneider (2011).

7 "The Blue Book was removed from circulation as an official act to consciously remove a critical account of the German colonial period of Namibian history. Copies of the report were destroyed with the aim of achieving reconciliation within the white settler community between the remaining German community and a new wave of, mainly Afrikaner, settlers. African voices were forgotten and their written statements actively erased." (Silvester \& Gewald 2003: xiii-xiv) 
cubiertas por un mantel negro cada una. Allí se da el encuentro entre palabra y texto, entre los cuerpos que leen y los documentos que sobrevivieron la destrucción. Entre las mesas y el público, hay un espacio vacío que permite la distancia entre el espacio del escenario y la audiencia, la cual marca asimismo la distancia teatral. Del otro extremo del escenario, la audiencia funciona como un Tribunal, la cual, como quiero exponer acá, se extiende temporal y espacialmente a través de la grabación de la performance y a partir de su re-exposición al seno de diversas audiencias.

Esta idea de la distancia teatral propia de la constitución de la audiencia como un Tribunal se produce gracias al intercambio entre la distancia temporal de la documentación y el marco de la actualización de esa distancia en la lectura de los archivos, así como en la continua re-actualización de la performance a través del video.

La escena que incluye a la audiencia se encuentra atravesada por varios niveles de documentación que involucran diversos procesos de memoria cultural (Assmann 2008) y postmemoria (Hirsch 2008). En primer lugar, la edición de los documentos sobre el genocidio en Namibia. En segundo lugar, las formas en que interactúan la memoria particular, la cual se encuentra atravesada por otros insumos de la memoria cultural como libros, fotografías y demás documentos, y también por la ausencia de estos registros, por el olvido como componente de la memoria. Esta confrontación que atraviesa el espacio escénico tradicional se extiende hacia la audiencia y abre el horizonte de la performance a otros espacios y tiempos, como se da con la grabación de la performance y su futura re-exposición.

En el Prefacio de su libro The bodies that were not ours, Coco Fusco (2001) justifica cuál es su interés por relatar y volver sobre los recuerdos y las historias de sus familiares, "I do so because the story represents my personal link to a very political history of colonialism, and that history has shaped a very specific relationship between mind and body for colonized and enslaved peoples" (xiv).

Tanto en la performance realizada en la Sophienscele, como en la narrativa de Fusco, la cuestión de la performatividad del documento aparece como un aspecto neurálgico de sus trabajos, mostrando precisamente las formas bajo las cuales se mediatizan las estructuras de una historia reciente y no tan reciente con los esquemas afectivos actuales, lo cual permite entender las dificultades de separar archivo y performance bajo el esquema de una ontology of liveness (Auslander 1999) que piensa la actualidad bajo los límites de la inmediatez y la fugacidad.

\section{Performing the Archive}

La oposición entre performance y archivo ha funcionado como un parte aguas al interior de los Performance Studies, pues mientras algunos afirman que la performance se define por la inmediatez y la fugacidad, otros sostienen que la Performance es la casa de la repetición performativa (Schneider 2011: 108).

En el primer caso, el capítulo séptimo del texto de Peggy Phelan (1996) Unmaked, “The ontology of performance: representation without reproduction” representa el ejemplo más explícito. En éste, las formas del archivo, por ejemplo, la grabación de una performance, son consideradas como la antítesis de la performance, pues la performance es pensada como lo inaprensible, lo evanescente, lo que resiste a la circulación de bienes dentro de la economía capitalista. En palabras de Peggy Phelan: 
Performance's only life is in the present, cannot be saved, recorded, documented, or otherwise participate in the circulation of representations of representations [...] The document of a performance then is only a spur to memory, an encouragement of memory to become present (146).

Performance resists the balanced circulations of finance. It saves nothing; it only spends (148).

Para Schneider, quien piensa la relación archivo-performance bajo un esquema de articulación continua, la noción de performance de Peggy Phelan lejos de resistir esa lógica del archivo, participa de ella al no considerar otras formas de "permanencia" (remains) en la esfera del performance. Schneider ubica la posición de Phelan dentro del contexto museístico que discute sobre la posibilidad de pensar un reducto que no pueda ser cooptado por la lógica del archivo, afirmando una perspectiva del archivo como objeto clausurado. Tal como lo propone Boris Groys (1992), la lógica de lo nuevo funciona gracias al intercambio entre el espacio profano y el sagrado que constituye el orden del archivo. El archivo vendría a incluir dentro de su lógica elementos que se encontraban dentro del espacio profano de la vida bajo el marco del espacio sagrado del archivo. Con este gesto de desplazamiento y apropiación, lo nuevo aparece como tal gracias a su inscripción en el archivo, provocando su próxima anulación. El rechazo a considerar el vínculo bilateral entre performance y registro rechaza ad portas la actividad performativa del archivo, al ser éste identificado como espacio mortuorio.

Al contrario de esta perspectiva, la performance de Coco Fusco permite desafiar la noción del documento como mero estímulo para la memoria al escenificar una lectura de documentos que han servido en otros momentos como herramienta política entre los poderes coloniales británico y alemán, y que son usados en la Sophienseele en Berlín casi un siglo después como documentos que performan la marca del colonialismo como repetición. Esta obra de Coco Fusco señala justamente la capacidad performativa de los archivos y la capacidad de archivar de la performance. En The Couple in a Cage ya se había mostrado cómo el archivo no funciona como superación y borramiento, pues el video también funcionaba como una producción constitutiva del evento en vivo y en registro.

Para entender esto hay que poner en entredicho la noción de referencia ${ }^{8}$ y reapropiación bajo el esquema de adecuación y fidelidad ${ }^{9}$ que subyace al reclamo entre performance y grabación para entender cómo funciona el archivo como medio de desplazamiento que hace intervenir elementos que se creían superados con lo contemporáneo: ni el performance es un evento efímero y homogéneo, ni el archivo es un evento clausurado.

Otro elemento importante reside en el concepto de memoria que liga los eventos con formas de imaginar el pasado en relación con distintas perspectivas sobre la actualidad. En este sentido, lo que se presenta en la performance no tiene que ver con lo que realmente pasó, sino con las formas en que eso que pasó vuelven a reinstalarse en otras esferas de la vida pública performando

8 Sobre esto, véase el análisis de Rancière (2011) en "Si existe lo irrepresentable".

9 Erika Fischer-Lichte, quien a pesar de parecer querer solventar la fuerte oposición en la cual se encuentra la apreciación de Phelan, también termina por afirmar que lo que ocurre en la realización escénica no puede reproducirse fielmente. La diferencia entre performance y documento que plantean ambas autoras supondría que habría una "actualidad" (gegenwärtig) en la materialidad de la realización escénica que no puede reproducirse, en el sentido en que la espacialidad, la sonoridad, por ejemplo, estarían lejos de toda documentación fiel. 
justamente lo que queda (remains) dentro de las prácticas del presente. Esto implica además plantear otra concepción de la memoria en relación con su carácter creativo (Feierstein 2012).

Por otro lado, esta cuestión de la no fidelidad del documento al performance se encuentra además como parte de las cuestiones que Coco Fusco pareciera introducir desde el título de su Performance, Words May Not Be Found. La versión crítica editada por Jeremy Silvester y Jan-Bart Gewald (2003) de The Blue Book fue intitulada Words Cannot be found. Este título proviene del testimonio que dio Jan Kubas, quien fue un testigo ocular de la derrota de los Hereros en la batalla de Hamakari en 1904. Trece años después de los hechos, Kubas afirma "Words cannot be found to relate what happened; it was too terrible" (citado por Silvester \& Gewald 2003: xiii). Según este testimonio, no solamente el horror de los hechos enmaraña la descripción adecuada de lo sucedido, sino que además encontrar las palabras supone también hurgar en los recuerdos que han sido transformados por el paso del tiempo, lo que supone el carácter de reescritura constante del trabajo de la memoria. El título de la performance de Coco Fusco pone además el acento sobre la posibilidad de encontrar o no las palabras. La transformación del "cannot" al "may not" señala un espacio de indescernibilidad y, desde mi punto de vista, un señalamiento al carácter de futuro que conlleva esta compilación de textos sobre el genocidio en Namibia.

Esta apertura de lugares de la cual participa la performance de Coco Fusco expone estos documentos en el seno de una sociedad donde este genocidio no ha tenido un proceso de rememoración colectiva sobre ese genocidio como sí ha sucedido respecto de lo ocurrido durante la Segunda Guerra Mundial. La postmemoria, tal como la entiende Hirsch, es una estructura generacional arraigada a formas de mediación y no una posición de identidad. ${ }^{10} \mathrm{El}$ "trabajo postmemorial"11 en esta performance tiene que ver también con reunir esas generaciones que no han estado vinculadas inmediatamente a la memoria del genocidio en Namibia para discutir cómo se entiende este trabajo post-memorial en el contexto alemán.

Coco Fusco está presentando esta performance en Berlín, donde, tal como quedó claro en la discusión que se abrió al final ${ }^{12}$ de la performance, muchos de los que estábamos allí no sabíamos mucho o casi nada acerca del genocidio en Namibia. Algunos de los presentes reclamaron el hecho de que en el sistema escolar nunca se habló de ello. El trabajo postmemorial pasa así por una "cultural memory" (Assmann 2008), es decir, por la construcción de sistemas simbólicos, que incluyen tanto esta performance como otros tipos de "symbolic systems" (Hirsch 2008: 110) y que van a permitir este trabajo postmemorial gracias a la "puesta en archivo" (Ricœur 2004: 215) de diferentes tipos de documentos, lo cual entra también dentro de las formas que materializan la interacción entre propuesta escénica y audiencia presentada al inicio de este texto.

Esta discusión también puede extenderse a las exposiciones documentales de Museos, donde éstos lejos de ser únicamente procesos neutrales de exhibición de objetos, exhiben la capacidad

10 "Postmemory is not an identity position but a generational structure of transmission deeply embedded in such forms of mediation" (Hirsch 2008: 114).

11 "Postmemorial work, I want to suggest—and this is the central point of my argument in this essay-strives to reactivate and reembody more distant social/national and archival/cultural memorial structures by reinvesting them with resonant individual and familial forms of mediation and aesthetic expression. Thus less-directly affected participants can become engaged in the generation of postmemory, which can thus persist even after all participants and even their familial descendants are gone" (Hirsch 2008: 111).

12 La discusión final era parte, desde mi punto de vista, constitutiva de la performance. 
de poner en escena debates que vuelven a pensar los procesos de representación de los hechos, relocalizándolos en la escena pública. Tal es el caso de la exposición que inauguró el Deutsches Historisches Museum el 14 de octubre del año 2016, Deutsches Kolonialismus. Fragmente seiner Geschichte und Gegenwart ${ }^{13}$, la cual estuvo abierta al público hasta el día 14 de mayo del año 2017. La performance de Coco Fusco coincide con esta gran exposición en el Museo de Historia de Berlín. Tal como lo indica Ulrike Kretzschmar en el prefacio al catálogo de la exposición,

for a long period Germany's colonial history occupied a subordinate place in the memorial culture of the Federal Republic of Germany. Increasing public awareness and a growing of debate in society about the German colonial past have become discernible only in recent years (Deutsches Historisches Museum 2016: 10)

El colonialismo alemán y particularmente el genocidio de las tribus de Herero y Nama es el acontecimiento que comparten estas dos muestras, a saber, el performance y la exposición museística. La coincidencia de estas dos exposiciones nos señala ya la apertura de un contexto institucional de cuestionamiento de las formas de memoria y de la capacidad que tienen los archivos para abrir y performar algunos fragmentos de la historia que de alguna manera no han tenido la mediación que otros sí han gozado. El trabajo postmemorial pasa en este sentido por la puesta en escena de esta memoria cultural, sin la cual una memoria del genocidio de Namibia no existiría dentro del contexto alemán. Se trata de un trabajo entonces sobre la memoria no de las víctimas, sino sobre las generaciones que posiblemente no han tenido ningún contacto con esa historia, pero que se encuentran vinculadas en términos de su pertenencia a una Nación que es el resultado cultural, político y económico de las relaciones inter y extra territoriales. Es decir que el olvido y el no reconocimiento de lo sucedido no implican la inexistencia de formas que son efectos de lo sucedido y de figuras no conscientes del genocidio en Namibia por parte de las fuerzas coloniales alemanas.

\section{Ontology of liveness}

Teniendo en cuenta estos elementos, resulta importante rescatar otros argumentos que han señalado la importancia de no oponer la performance al documento. Philip Auslander (1999) critica la "ontology of liveness", apuntando a cuestionar la noción de inmediatez que se supone existe en las performances y su oposición intrínseca a lo mediático. Auslander argumenta que existe el supuesto generalizado según el cual "the live event is 'real' and that mediatized events are secondary and somehow artificial reproductions of the real" (3). Existiría un prejuicio que ubica al teatro y a los "live performance" en una lógica distinta al de la economía de mercado y a la de los medios masivos (mass media), mientras que, para él, se trata de una "competitive opposition at the level of cultural economy" (11); a saber, se trata de un problema de valoración y de intercambio cultural, y no de un problema ontológico. Introduce la importancia de entender las operaciones lógicas dentro de términos culturales y no como una oposición respecto de la naturaleza de los objetos. 
Esto implica que habría que iniciar por pensar los marcos conceptuales bajo los cuales establecemos la diferencia entre estos ámbitos, pues esto nos revelaría que lejos de plantear la naturaleza intrínseca del "archivo" o del "performance”, lo que hacemos es referirnos a una jerarquía de valores.

En este sentido, las perspectivas sobre los registros de las performances tienen que ver con ciertos supuestos que se estarían planteando: en primer lugar, el supuesto de que existe una inmediatez de la performance y, en segundo lugar, la idea según la cual una realización escénica tiene una constitución homogénea en el momento en que se desarrolla in situ, como si esa realización, lo que sea esta realización, no dependiera también de las diferentes experiencias que puedan tenerse de ella, es decir, de las formas de estar en la performance, de los diferentes puntos de vista que sin duda alguna constituyen la performance. Puntos de vista que se traducen en los documentos, ciertamente, a partir de otra lógica, la cual tiene que ver con la cámara y los dispositivos de reproducción, pero que no descartan que en el proceso de una performance también existen niveles de mediación y que en una grabación también hay una performance, aunque no sea la misma. Tendríamos más bien que pensar en términos fenomenológicos de cómo se muestran (show) y se relacionan los elementos de una determinada performance, y no afirmar que la performance existe como un dato en el sentido de la actitud natural que critica Husserl respecto del tratamiento del "mundo" como un dato dado de antemano claro y cierto.

En este sentido, la oposición que se ha establecido al interior de los Performance Studies no cuestiona el sistema de valores sobre el cual se funda la presentación de la performance como algo inmediato y dado por sí mismo. Por el contrario, esta perspectiva revitaliza los patrones de una estética fundada en lo auténtico o lo original, entendiendo el "origen" como lo anterior a otra cosa de la cual es fundamento. La memoria y los documentos que la estimulan como apuntan Auslander y Schneider, en la medida en que vuelven a aparecer, funcionan desde su capacidad medial y performativa; respondiendo a plataformas históricas de exhibición, a la historia misma de sus producciones y no a un supuesto carácter intrínseco de "lo en vivo". Todo, si se quiere, se encuentra en vivo; también el archivo al ser inscrito en formas de uso.

En este sentido, la pregunta sobre el lugar de la performance respecto del archivo tendría que plantearse sobre el tipo de performance que las "documentaciones" realizan y con ello discutir los límites respecto de la expansión de este "campo" de la performance. Como apunta Rebecca Schneider,

If we consider performance as of disappearance, of an ephemerality read as vanishment and loss, are we perhaps limiting ourselves to an understanding of performance predetermined by our cultural habituation to the logic of the archive? According to the logic of the archive, what is given to the archive is that which is recognized as constituting a remain, that which can have been documented or has become document. (2011: 98).

A partir de Schneider y del epígrafe que abre este texto, al afirmar esta oposición entre documento y performance, ¿se estarían ignorando otras formas de conocer, recordar, que nos indicarían el espacio en el cual "performance remains"; formas que no parten de la lógica de la mimesis, de la adecuaetio y de la referencialidad; formas en donde el performance no se opondría a la historia, la memoria, las tradiciones orales o la presencia de estas en los gestos o el movimiento de los cuerpos? 


\section{Conclusiones}

El archivo, como afirma Arlette Farge "est excess de sens" (Farge 1989: 42) y se sitúa, desde la perspectiva de Georges Didi-Huberman (2000), como espacio de interrogación que hace converger los tiempos. Los testimonios que han sido leídos en la Sophiensaele y que yo he revisitado a través del video, permiten traer esas historias al presente y nos demandan (claim) una apertura de nuestra parte para ser escuchados. Como afirma Derrida, lo que distingue un testimonio de la simple transmisión de conocimiento es que

quelqu'un s'engage auprès de quelqu'un, par un serment au moins implicite. Le témoin promet de dire ou de manifester à autrui, son destinataire, quelque chose, une vérité, un sens qui lui a été ou qui lui est de quelque façon présent, à lui-même en tant que témoin -seul et irremplaçable (2005: 45-46).

Quienes acompañan a Coco Fusco en el escenario son testigos de los testimonios que han sido publicados en The Blue Book, el cual es una prueba documental del genocidio que tuvo lugar en Namibia. Constituye, desde el punto de vista del historiador, como afirma Paul Ricœur, una "puesta en archivo". Estos textos son ahora puestos en escena por Coco Fusco después de pasar por varios esquemas narrativos (el contexto que da nacimiento al texto y su re-edición crítica por Silverst y Gewald) y son ahora escuchados, quizá, por primera vez por algunos de nosotros. Los fragmentos del libro son presentados desde otra materialidad: la voz de quienes nos los leen, el escenario en el cual los leen y en el cual nos encontramos en ese momento y, desde la grabación a partir de la cual he accedido de nuevo a esta performance que no deja de ser una performance, según he querido sostener aquí en relación con el campo expandido.

Tal como se dijo, en la narrativa de los textos de Coco Fusco también encontramos un fuerte interés por pensar los cuerpos como lugar de archivo de lo colectivo. Tal como lo expone Cathy Caruth (2016) respecto del trauma, es en la experiencia individual donde se encuentran lo colectivo y lo político, y la pregunta por el quién habla se inscribe en el cruce entre las memorias que se localizan en el cuerpo de quien habla, como locus, pero no como origen y fin de los discursos.

En el cuerpo de la performance de Coco Fusco se cruzan esas historias colectivas e individuales y en este sentido es justamente allí donde se conecta lo individual con lo político y lo colectivo y donde se performa el archivo.

Este compromiso del testigo y del escucha inscribe no solamente la promesa, sino que también localiza un campo teatral de enunciación que se abre gracias a los distintos medios de enunciación y nos plantean la pregunta de Cathy Caruth que se ha colocado en el epígrafe. "What do the dying bodies of the past [...] have to do with the living bodies of the present? And what is the role of our seeing in establishing a relation between these two sets of bodies?" (2016: 26-27).

Cathy Caruth sostiene que es justamente esta latencia entre lo conocido y lo no conocido donde se da el "political bond to other histories. To put it somewhat differently, we could say that the traumatic nature of history means that events are only historical to the extend that they implicate others" (2016: 19-20). Es justamente en esta apertura hacia la escucha que demandan las palabras en esta performance y que se actualiza en cada una de las proyecciones, la que permite entender que los archivos (se) performan y que la performance también queda (remains). 


\section{Referencias bibliográficas}

Assmann, J. (2008). Communicative and Cultural Memory. In A. E. Erli \& A. Nünning (Eds.), Cultural Memory Studies. An International and Interdisciplinary Handbook (pp. 109-118). Berlin, New York: Walter de Gruyter.

Auslander, P. (1999). Liveness: Performance in a Mediatized Culture. London and New York: Routledge.

Caruth, C. (2016). Unclaimed Experience: Trauma, Narrative and History. Baltimore: Johns Hopkins University Press.

Derrida, J. (2005). Poétique et politique du témoignage. Paris: L’Herne.

Didi-Huberman, G. (2000). Devant le temps. Histoire de l'art et anachronisme des images. Paris: Les Éditions de Minuit.

Farge, A. (1989). Le goût de larchive. Paris: Éditions du Seuil.

Feierstein, D. (2012). Memorias y representaciones. Sobre la elaboración del genocidio. Buenos Aires: FCE.

Fusco, C. (1988). The Other History of Intercultural Performance. TDR, 38 (1), 143-167.

Groys, B. (1992). Du nouveau. Essai d’économie culturelle. Nîmes: Éditions Jacqueline Chambon.

Halbwachs, M. (1997). La mémoire collective. Paris: Albin Michel.

Hirsch, M. (2008). The Generation of Postmemory. Poetics Today, 29 (1), 103-128. https://doi. org/10.1215/03335372-2007-019

Krauss, R. (1979). Sculpture in the Expanded Field. Spring, 8, 30-44.

Museum, D. H. (2016). German Colonialism. Fragments Past and Present. Berlin: Deutsches Historisches Museum.

Phelan, P. (1996). The ontology of performance: representation without reproduction. In Unmarked. The politics of performance (pp. 146-166). New York - London: Routledge.

Rancière, J. (2011). El destino de las imágenes. Buenos Aires: Prometeo Libros.

Ricœur, P. (2004). La memoria, la historia, el olvido. Buenos Aires: FCE.

Schneider, R. (2011). Performing Remains. Art and war in times of theatrical reenactment. London, NY: Routledge.

Silvester, J.; \& Gewald, J.-B. (Eds.). (2003). Words Cannot Be Found. German Colonial Rule in Namibia. An Annotated Reprint of the 1918 Blue Book. Leiden, Boston: Brill.

Taylor, D. (2003). The archieve and the repertoire (3a ed.). North Carolina: Duke University Press.

. (2009). Performance e historia. Apuntes, 131, 105-123. 